\title{
Intervención telefónica y competencia para cuidar de personas con enfermedad crónica
}

\author{
Telephone intervention and proficiency to take care of people with a chronic \\ disease
}

Recibido:

25 Septiembre de

2017

Aprobado:

22 Noviembre 2017
Autor de correspondencia

* $\square$ Doctora en Enfermería. Magister en Enfermería. Profesora Asociada Universidad Nacional de Colombia Correo: gmcarrillog@unal.edu.co. Orcid: 0000-00034513-104X

** Enfermera. Magister en Análisis sistémico. Joven Investigador Colciencias. lagonzalezv@unal.edu.co. Orcid: 0000-0002 7597-8025

Financiación: Convocatoria Joven Investigador Colciencias 2016-2017.

\section{Intervenção telefônica e habilidade para cuidar de pessoas com doença crônica}

\author{
Gloria Mabel Carrillo González* \\ Lizeth Adriana González Vega**
}

\section{Resumen}

Objetivo: Determinar el efecto de una intervención telefónica en personas con enfermedad crónica para mejorar su competencia de cuidado en el hogar. Materiales y Métodos: Estudio descriptivo y exploratorio; se vincularon 37 personas con enfermedad crónica, definidas como casos, que ingresaron a una institución de salud de la ciudad de Bogotá. Se aplicó la intervención telefónica durante 1 mes después del egreso hospitalario, se realizó medición antes y después de la competencia para el cuidado en el hogar y se registraron cada uno de los seguimientos telefónicos para determinar la percepción de los usuarios frente a los mismos. Resultados: La mayoría de pacientes son de sexo masculino, con diagnósticos de hipertensión arterial, diabetes mellitus tipo II, enfermedad pulmonar obstructiva crónica- EPOC, enfermedad renal e insuficiencia cardíaca, una edad promedio de 66 años y ocupación hogar. El $100 \%$ de la muestra tiene más de una enfermedad crónica y bajos niveles de dependencia. La competencia para cuidar reportó niveles medios previo al inicio de la intervención, al finalizar aumentó, con cambios estadísticamente significativos. En cuanto a la percepción, se reconoce esta intervención como una continuidad de la atención, surgen temas de interés sobre la regulación del consumo de agua, la administración de medicamentos y la dieta y se demanda el abordaje de los componentes emocionales, sociales y económicos. Conclusiones: La intervención telefónica es una herramienta eficaz para aumentar la competencia de cuidar en el hogar de las personas con enfermedad crónica, genera percepción de seguridad, continuidad y vínculo con el equipo de salud.

Palabras clave: Pacientes, alta del paciente, enfermedad crónica

\section{Abstract}

Objective: To determine the effect of a telephone intervention in people with a chronic disease to improve their proficiency of care at home. Materials and Methods: Descriptive and exploratory study; 37 people with a chronic disease were enrolled, defined as cases, that came in to a health institution in Bogota. The telephone intervention was applied for a month after leaving the hospital, measurement of proficiency was taken before and after for care at home and each telephone followup was registered to determine the perception of the users toward this intervention. Results: The majority of patients are males, with diagnoses of arterial hypertension, diabetes mellitus type II, chronic obstructive pulmonary disease- COPD, kidney failure and congestive heart failure, an average age of 66 years and domestic-work dedication. 100\% of the sample has more than one chronic disease and low levels of dependency. The proficiency to take care of, demonstrated medium levels before the beginning of the intervention, at the end it increased with significant statistic changes. Regarding perception, this intervention is recognized as a continuity of care, relevant

\section{Para citar este artículo/ To reference this article/ Para citar este artigo/}

Carrillo Gonzalez GM, Gonzalez Vega LA. Intervención telefónica y competencia para cuidar de personas con enfermedad crónica. Rev. cienc. cuidad. 2018;15(1):98-109

Este es un artículo bajo la licencia CC BY (https://creativecommons.org/licenses/by/4.0/) @ ) 
topics come up about the regulation of water consumption, medication administration and the diet, and an approach to emotional, social and economic components is demanded. Conclusions: The telephone intervention is an effective tool to increase the proficiency of care at home for people with a chronic disease, it generates a perception of safety, continuity and a bond with the health care team.

E-ISSN 2322-7028

Vol. 15 No. 1

Keywords: Patients, patient discharge, chronic disease

\section{Resumo}

Objetivo: Determinar o efeito de uma intervenção telefônica em pessoas com doença crônica para melhorar sua habilidade de cuidado no lar. Materiais e Métodos: Estudo descritivo e exploratório; se vincularam 37 pessoas com doença crônica, definidas como casos, que ingressaram a uma instituição de saúde da cidade de Bogotá. Aplicou-se a intervenção telefônica durante 1 mês depois da saída hospitalar, se realizou medição antes e depois da habilidade para o cuidado no lar e se registraram cada um dos seguimentos telefônicos para determinar a percepção dos usuários frente aos mesmos. Resultados: A maioria de pacientes são de sexo masculino, com diagnósticos de hipertensão arterial, diabetes mellitus tipo II, doença pulmonar obstrutiva crônica- EPOC, doença renal e insuficiência cardíaca, uma idade média de 66 anos e ocupação no lar. O $100 \%$ da amostra tem mais de uma doença crônica e baixos níveis de dependência. A habilidade para cuidar reportou níveis médios prévio ao início da intervenção, ao finalizar aumentou, com câmbios estatisticamente significativos. Enquanto à percepção, se reconhece esta intervenção como uma continuidade do atendimento, surgem temas de interesse sobre a regulação do consumo de água, a administração de medicamentos e a dieta e se demanda a abordagem dos componentes emocionais, sociais e econômicos. Conclusões: A intervenção telefônica é uma ferramenta eficaz para aumentar a habilidade de cuidar no lar das pessoas com doença crônica, gera percepção de segurança, continuidade e vínculo com a equipe de saúde.

Palavras-chave: Alta do paciente, doença crônica, pacientes

\section{Introducción}

Las enfermedades crónicas están consideradas actualmente como una epidemia mundial y como la principal causa de mortalidad en el mundo contemporáneo, siendo responsables del $68 \%$ de los 56 millones de defunciones registradas en 2012, de acuerdo con lo reportado por el Informe sobre la Situación Mundial de las Enfermedades No Transmisibles 2014 (1,2).

Este tipo de enfermedades comparten características comunes, pese a que tradicionalmente han sido estudiadas de forma aislada y abordadas por los servicios asistenciales de manera atomizada. Dentro de tales características se encuentra que se agregan unas a otras potenciando el deterioro orgánico y la afectación funcional de los pacientes, su etiología es multicausal y compleja, su aparición es gradual y silente, su pico de prevalencia aparece en edades avanzadas (3), disminuyen la calidad de vida de los enfermos afectados, son motivo de muerte prematura y causan efectos económicos importantes en las familias y en la sociedad en general (4), además de requerir de largos períodos de cuidado, tratamiento paliativo y seguimiento permanente por parte de los profesionales de la salud (5).

Dicho esto, es posible también establecer otro punto de observación para las intervenciones en salud, no sólo sobre 'la enfermedad crónica' (EC) sino sobre la experiencia o la situación de cronicidad, que incluye los sentimientos de impotencia y frustración permanente de la persona que vive con enfermedad crónica, la pérdida de confianza en las capacidades de automanejo, el deterioro del funcionamiento laboral y de las relaciones familiares (6), una modificación obligada de los estilos de vida que tiende a persistir (7), un estado de indisposición producido por discapacidad o incapacidad que requiere alta intervención médico-social y que se deriva del proceso crónico, cuyas características tienen raíces profundas en los significados subjetivos que los rodean (8) y un proceso de trasformación de la vida cotidiana que está en estrecha relación con símbolos y representaciones socialmente construidas, sostenidas, además, en una fuerte carga valorativa (9). 
ISSN-PRINT

1794-9831

E-ISSN 2322-7028

Vol. 15 No. 1

Ene - Jun 2018

Cúcuta, Colombia
De acuerdo con lo anterior, pensar la EC, pero además pensar la situación de cronicidad, implica ampliar la mirada más allá del tratamiento, dando lugar a la observación de la dimensión subjetiva y experiencial que rodea a los enfermos (10).

En este contexto, los programas de transición y seguimiento son estrategias que permiten constituir una mirada más compleja que da lugar a la observación de estas dos 'caras': enfermedad crónica más situación de cronicidad, y, además, de acuerdo con la experiencia investigativa presentada aquí, ofrece la oportunidad de realizar una mirada crítica y propositiva sobre todo el proceso de atención de enfermería en el contexto clínico (11).

Ahora bien, en el contexto colombiano, la Política Integral de Atención en Salud (PAIS) invita a los actores del Sistema de Salud a generar abordajes integrales de las necesidades de salud de las personas con enfermedades crónicas y las familias, con estrategias de intervención individual y en poblaciones de riesgo. Al considerar dicha política se comprueba la prioridad que representa intervenir en salud pública con programas que, en un corto plazo, aporten de forma concreta al componente operativo en el Modelo de Atención Integral en Salud (MIAS), con el fin de garantizar que los ciudadanos reciban servicios seguros, accesibles y humanizados (12).

Por otro lado, el abordaje integral de las personas con enfermedades crónicas implica contar con programas que favorezcan la disponibilidad de los servicios, disminuyan los costos generales y contengan elementos de cuidado en el hogar (13).

La gestión, a través de tecnologías de la información y la comunicación (TIC), se consolida como un método eficaz en el proceso de tratamiento de las EC (14). En tal sentido, las intervenciones telefónicas surgen como una tendencia válida que puede generar cambios en la actitud del paciente respecto a su condición, mejores comportamientos de automanejo e, incluso, impacto sobre el estado de salud y la calidad de vida.

Shearer et al. (15) señalan que el teléfono es una tecnología que está disponible para la mayoría de las personas, los sistemas de llamadas de salud que reciben los pacientes por parte de enfermería son rentables y se considera un método útil de tratamiento de la EC, generando un resultado terapéutico efectivo, el desarrollo de la comunicación enfermera-paciente y facilitando la superación de obstáculos de tiempo y distancia para proporcionar mejores cuidados (16). Por lo anterior, resulta de utilidad explorar el alcance de intervenciones telefónicas como estrategias para el abordaje integral de personas con EC. El objetivo del estudio fue determinar el efecto de una intervención telefónica para fortalecer la competencia de cuidado en el hogar de personas con enfermedad crónica después del egreso hospitalario en una institución de salud de Bogotá.

La competencia de cuidado se comprendió como la capacidad, habilidad y preparación que tiene la persona con enfermedad crónica para ejercer la labor de cuidar en el hogar. Por su parte, el concepto 'alta del paciente' se comprendió como el momento de egreso hospitalario efectivo.

\section{Objetivos}

\section{Objetivo general}

Evaluar el efecto de una intervención telefónica estandarizada para fortalecer la competencia de cuidado en el hogar en personas con enfermedad crónica en una institución hospitalaria en Bogotá.

\section{Objetivos específicos}

- Valorar la competencia para el cuidado en el hogar en un grupo de personas con enfermedad crónica y cuidadores familiares.

- Implementar un esquema de intervenciónseguimiento telefónico estandarizado en un grupo de personas con enfermedad crónica

- Revalorar la competencia para el cuidado en el hogar, una vez finalizada la intervención.

\section{Materiales y Métodos}

La investigación realizada corresponde a un diseño exploratorio con la metodología de estudio de casos. Es de señalar que la máxima prioridad de un estudio de casos consiste en generar conocimientos sobre el caso específico, permitiendo, en ocasiones, que se puedan examinar fenómenos que no han sido estudiados con el suficiente rigor (17). 
Los criterios de inclusión fueron: persona con enfermedad crónica diagnosticada, hospitalizada en el servicio de medicina interna, mayor de 18 años, en proceso de egreso hospitalario, con capacidad para comunicarse verbalmente y con acceso, conocimiento y posibilidad de uso de teléfono en el domicilio. Los criterios de exclusión fueron: pacientes en proceso de alta para programas de hospitalización domiciliario o con remisión a otra institución de salud.

Los casos fueron seleccionados de acuerdo con los criterios de inclusión, en los servicios de hospitalización de la institución de salud. Previo a la iniciación del estudio, todos los participantes firmaron y dieron su consentimiento informado.

Las variables estudiadas fueron: 1) Sociodemográficas: edad, sexo, nivel educativo, ocupación, estado civil y procedencia; 2) Clínicas: diagnóstico médico, estado mental, nivel de dependencia y competencia para cuidar en el hogar medida a través del instrumento CUIDAR $\AA$ versión abreviada que comprende 6 categorías: Conocimiento, Unicidad (condiciones personales), Instrumental, Disfrutar (bienestar), Anticipación y Relación social e interacción.

Este instrumento consta de 20 ítems que se miden mediante una escala tipo Likert y cuenta con las pruebas psicométricas para ser usado en Colombia. Frente a la validez de contenido, cuenta con un CVI global de 0.97 y frente a confiabilidad por consistencia interna obtuvo 0,96 medida con el alfa de Cronbach para esta aplicación $(10,18)$.

La recolección de datos se realizó entre enero y junio de 2017, mediante la captación de los casos en el servicio de hospitalización medicina interna. Se les explicaron los objetivos del estudio y el esquema de la intervención. Se realizó la aplicación de los instrumentos, se brindó información general sobre el tratamiento y acciones de cuidado en el hogar y se proporcionó material educativo de apoyo. Después del egreso se implementó la intervención telefónica y, un mes después del alta hospitalaria, se aplicó nuevamente la escala de competencia para el cuidado en el hogar. Adicionalmente, cada una de las percepciones de los participantes durante el seguimiento telefónico fue grabada, con su consentimiento.

La intervención telefónica retoma los elementos de la metodología del programa plan de egreso hospitalario y consiste en una serie de actividades coordinadas y ejecutadas por personal de enfermería. Se realizan 6 llamadas telefónicas, tal como se describe en la Tabla 1.

Tabla 1. Esquema de intervención telefónica en la transición hospital-hogar de personas con enfermedad crónica

\begin{tabular}{|c|c|c|c|}
\hline Llamada & $\begin{array}{c}\text { Tiempo después } \\
\text { del egreso }\end{array}$ & Objetivo & Duración \\
\hline 1 & 1 a 3 días & $\begin{array}{l}\text { Determinar cómo ha sido la transición del paciente a casa. } \\
\text { Brindar información sobre la disponibilidad del seguimiento telefónico. } \\
\text { Aclarar dudas sobre la transición }\end{array}$ & 15 minutos \\
\hline 2 & 4 a 7 días & $\begin{array}{l}\text { Brindar instrucción sobre manejo de medicamentos y dispositivos, signos de alarma para } \\
\text { consultar. } \\
\text { Indicaciones sobre dieta y alimentación, ejercicio y actividades de la vida diaria, } \\
\text { eliminación. }\end{array}$ & 15 minutos \\
\hline 3 & 8 a 13 días & $\begin{array}{l}\text { Brindar instrucción sobre higiene del sueño, manejo de síntomas, instrucciones específicas } \\
\text { de acuerdo a patología. } \\
\text { Refuerzo indicaciones dieta y ejercicio. } \\
\text { Aclarar dudas sobre la adaptación a la terapéutica. }\end{array}$ & 15 minutos \\
\hline 4 & 14 a 21 días & $\begin{array}{l}\text { Brindar instrucción sobre manejo de síntomas: dolor, fatiga, estreñimiento y otros referidos } \\
\text { por el participante. } \\
\text { Abordar el componente emocional. } \\
\text { Brindar espacio para aclarar dudas sobre trámites administrativos y consultas de control. }\end{array}$ & 15 minutos \\
\hline 5 & 22 a 28 días & $\begin{array}{l}\text { Brindar instrucción e información sobre manejo de síntomas referidos por el participante. } \\
\text { Explorar metas y planes de acción futuro con el participante. } \\
\text { Abordar el componente social y redes de apoyo primaria }\end{array}$ & 15 minutos \\
\hline 6 & 29 - 30 días & $\begin{array}{l}\text { Brindar información a las inquietudes referidas por el participante. } \\
\text { Aplicación de escala de competencia para el cuidado en el hogar } \\
\text { Aplicación de encuesta de satisfacción y percepciones frente al programa. }\end{array}$ & 15 minutos. \\
\hline
\end{tabular}

Fuente: Construcción propia de los autores, 2016 
ISSN-PRINT

1794-9831

E-ISSN 2322-7028

Vol. 15 No. 1

Ene - Jun 2018

Cúcuta, Colombia

\section{Análisis de datos}

Los datos fueron recogidos y procesados en Microsoft Excel 2012. Para la realización del análisis estadístico se utilizó el paquete SPSS versión 22.0, con Licencia de la Universidad Nacional de Colombia.

Para la comparación de la competencia de cuidado en el hogar de los casos antes y después de la intervención se acudió a una prueba de tipo no paramétrico. Se recurrió al análisis de contenido de los textos resultado de la grabación de las llamadas telefónicas para describir la percepción de los participantes frente a la intervención.

\section{Consideraciones éticas}

Dentro de las consideraciones éticas se tuvieron en cuentalaspautaséticas paralainvestigaciónbiomédica preparadas por el Consejo de Organizaciones Internacionales de las Ciencias Médicas -CIOMS(19) y los parámetros establecidos en la Resolución 8430 de 1993 expedida por el Ministerio de Salud de Colombia (20).

Se contó con el aval del Comité de la Facultad de Enfermería de la Universidad Nacional de Colombia.

\section{Resultados}

\section{Características de los participantes}

Los casos corresponden a personas con enfermedad crónica, con edades entre los 22 y 95 años (con un promedio de 66 años), entre quienes predomina el género masculino, ocupación hogar, estado civil casado y procedencia urbana. Frente al nivel de escolaridad, los datos fueron heterogéneos: se encontró que el $20 \%$ de los participantes cuenta con primaria completa, el $20 \%$ con bachillerato completo y el 20 $\%$ con nivel universitario (el porcentaje restante se distribuye entre personas analfabetas, con primaria o bachillerato incompleto, técnicos y con formación de posgrado). Dentro de la morbilidad reportada se encuentra predominio de la hipertensión arterial, diabetes mellitus tipo II, EPOC, enfermedad renal e insuficiencia cardíaca. El $100 \%$ de los participantes tiene más de una enfermedad crónica y bajos niveles de dependencia (el $72 \%$ cuentan con un puntaje PULSES ${ }^{1}$ entre 6-8). (Ver Tabla 2)

Tabla 2. Características de los casos

\begin{tabular}{lll}
\hline & Femenino & Masculino \\
\hline Número de participantes & 12 & 25 \\
Edad promedio & 66 & 68 \\
Ocupación & Hogar & Pensionado \\
Estado civil predominante & Casada-Viuda & Casado \\
Escolaridad predominante & Primaria Incompleta & Primaria completa \\
Diagnostico principal & Hipertensión arterial & Hipertensión arterial- diabetes mellitus \\
Nivel de dependencia & Bajo (75 \%) & Bajo (70\%) \\
Escala SPMSQ & Normal (91\%) & Normal (100 \%) \\
\hline
\end{tabular}

Fuente: Datos del estudio, 2017

Resultado de la aplicación de la Escala que lleva el mismo nombre y mide: Estabilidad de la patología, utilización de miembros superiores, capacidad de locomoción, capacidad sensorial, eliminación y actividad social. 


\section{Competencia para el cuidado en el hogar}

Antes de la intervención, el nivel de competencia para el cuidado en el hogar de los pacientes obtuvo una media de 45,86, desviación estándar (DS) 7,7.

Las dimensiones que obtuvieron medias con menor puntuación antes de la intervención telefónica fueron conocimiento y anticipación. La dimensión conocimiento indaga por la información que conoce la persona con enfermedad crónica frente a su diagnóstico, plan de tratamiento y signos de alarma, y la anticipación sobre las capacidades frente a los riesgos que para su salud implica su condición de cronicidad y en la organización de los recursos necesarios para el cuidado.

Sobre estas dos dimensiones los pacientes refieren conocimiento escaso o impreciso frente a las complicaciones que pueden derivarse de su situación de cronicidad, la baja adherencia a los medicamentos o la pobre regulación del patrón dietético (por ejemplo, pacientes con HTA o diabetes no tienen conocimiento de la estrecha relación de estos dos diagnósticos con el riesgo de desarrollar insuficiencia renal crónica).

Por su parte, las dimensiones que obtuvieron mayores puntajes antes de la intervención telefónica fueron unicidad (condiciones personales) y relación social e interacción. La dimensión unicidad explora la capacidad de cuidarse a sí mismos y contar con un plan de vida a pesar de la situación de cronicidad. La dimensión relación social e interacción indaga por los vínculos y relaciones familiares, así como por la capacidad de recibir apoyo de otros para el propio cuidado.

El mayor puntaje obtenido en estas dimensiones se relacionó con la postura personal de los pacientes para asumir que hay que "acostumbrarse" a vivir con la enfermedad crónica y que ella no debería constituir una barrera para llevar adelante los planes personales (estudio, trabajo, cuidado de los hijos, etc.)

Además, los participantes resaltaron el rol determinante del apoyo familiar no sólo durante el proceso de hospitalización sino en el hogar: recordando y acompañando a los controles y exámenes médicos, brindando apoyo económico y emocional e, incluso, uniéndose a los cambios en el estilo de vida que exige la enfermedad crónica -algunos refirieron, por ejemplo, que ahora 'todos en la casa' dejaron de consumir grasa o le acompañan a hacer actividad física-.

Después de la intervención, se reportaron niveles de competencia más altos frente al inicio. En el análisis por dimensiones se identificó que los componentes de conocimiento, unicidad y disfrutar aumentaron, siendo estos cambios estadísticamente significativos (p 0,001). (Tabla 3). Figura 1.
ISSN-PRINT

1794-9831

E-ISSN 2322-7028

Vol. 15 No. 1

Ene - Jun 2018

Cúcuta, Colombia 
ISSN-PRINT

1794-9831

E-ISSN 2322-7028

Vol. 15 No. 1

Ene - Jun 2018

Cúcuta, Colombia

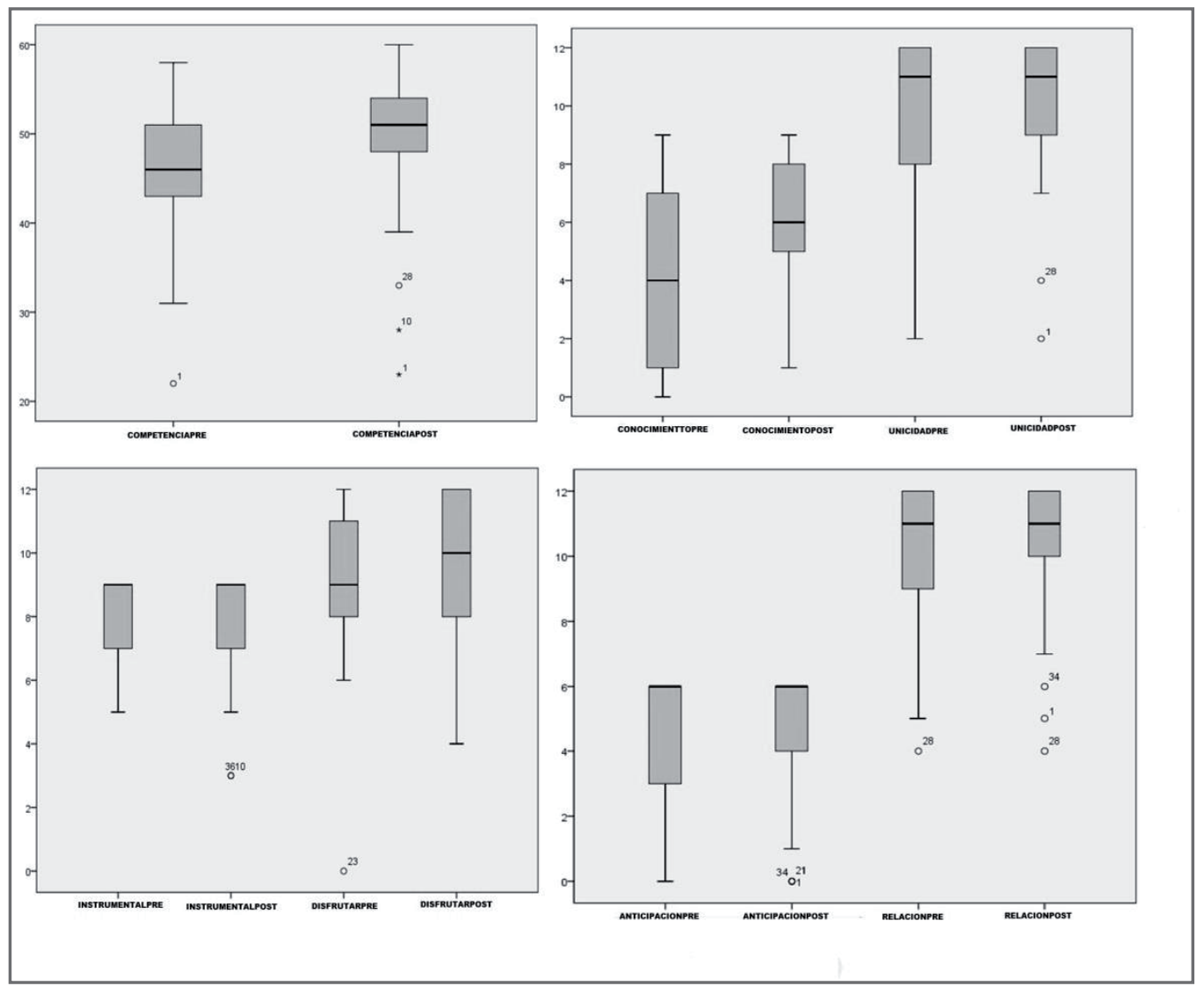

Figura 1. Diagrama de cajas. Competencia para el cuidado en el hogar antes y después de la intervención

Fuente: Datos del estudio, 2017.

Este hechose puede relacionar con dos componentes claves del seguimiento telefónico instaurado: 1) El desarrollo de instrucciones personalizadas y adaptadas al nivel educativo de los pacientes frente al manejo de la patología (medicamentos, dispositivos, dieta, actividad física) y los riesgos asociados (signos de alarma, identificación temprana de complicaciones, patologías asociadas); 2) La posibilidad que encuentra el paciente de plantear preguntas e inquietudes en cualquier momento del seguimiento, hecho que, además, se facilita por el contacto permanente que genera confianza con el profesional de enfermería que le acompaña. 
Tabla 3. Competencia para el cuidado en el hogar, reporte por dimensiones antes y después de la intervención telefónica

\begin{tabular}{|c|c|c|c|c|}
\hline \multicolumn{2}{|c|}{ VARIABLE } & \multirow{2}{*}{$\frac{\text { Media }}{45,9}$} & \multirow{2}{*}{$\begin{array}{c}\begin{array}{c}\text { Desviación } \\
\text { estándar }\end{array} \\
7,8\end{array}$} & \multirow{3}{*}{$\begin{array}{c}\begin{array}{c}\text { Prueba de } \\
\text { Wilcoxon }\end{array} \\
p 0,00\end{array}$} \\
\hline COMPETENCIA PARA & Antes de la intervención & & & \\
\hline CUIDAR & Después de la intervención & 49,2 & 7,9 & \\
\hline \multirow{2}{*}{ CONOCIMIENTO } & Antes de la intervención & 4,2 & 3,3 & \multirow{2}{*}{$p 0,00$} \\
\hline & Después de la intervención & 6,5 & 1,9 & \\
\hline \multirow{2}{*}{ UNICIDAD } & Antes de la intervención & 9,9 & 2,5 & \multirow{2}{*}{$p 0,026$} \\
\hline & Después de la intervención & 10,2 & 2,4 & \\
\hline \multirow{2}{*}{ INSTRUMENTAL } & Antes de la intervención & 7,9 & 1,4 & \multirow{2}{*}{$p 0,861$} \\
\hline & Después de la intervención & 7,8 & 1,7 & \\
\hline \multirow{2}{*}{ DISFRUTAR } & Antes de la intervención & 9,1 & 2,7 & \multirow{2}{*}{ p 0,001 } \\
\hline & Después de la intervención & 9,7 & 2,2 & \\
\hline \multirow{2}{*}{ ANTICIPACIÓN } & Antes de la intervención & 4,4 & 2,1 & \multirow{2}{*}{ p 0,109 } \\
\hline & Después de la intervención & 4,6 & 1,9 & \\
\hline \multirow{2}{*}{ RELACIÓN } & Antes de la intervención & 10,4 & 2,2 & \multirow{2}{*}{ p 0,180} \\
\hline & Después de la intervención & 10,4 & 2,2 & \\
\hline
\end{tabular}

Fuente: Datos del estudio, 2017
ISSN-PRINT

1794-9831

E-ISSN 2322-7028

Vol. 15 No. 1

Ene - Jun 2018

Cúcuta, Colombia

\section{Percepción de los usuarios frente a la intervención}

Se identificaron seis temas que dan cuenta de las percepciones de los participantes frente a la intervención y las necesidades de información de mayor interés.

\section{Acompañamiento telefónico, una continuidad} de la atención. Los 37 casos manifestaron una 'sorpresa' positiva al ser contactados por personal de la institución después del egreso para el seguimiento y lo asocian con una mayor calidad en el proceso de atención brindado. Se percibe, además, una noción de acompañamiento telefónico que les abre la posibilidad de que planteen preguntas sobre el proceso de tratamiento.

Ahora bien, se identificó incertidumbre sobre la temporalidad de la progresión de la enfermedad en todos los casos, situación que facilitó la comunicación con los participantes, ya que reconocían en este seguimiento un escenario para expresar sus inquietudes. Ahora bien, para los casos era llamativo que está intervención estuviese a cargo de un profesional de enfermería, quizás por desconocimiento del rol profesional para el egreso y como líder del seguimiento posterior.

\section{La regulación del consumo de agua, una pregunta} de interés. En el seguimiento telefónico se realizaron preguntas específicas en relación con la regulación del consumo de agua, especialmente en personas con insuficiencia cardíaca, enfermedad renal, EPOC y usuarios de nefrectomía. 30 casos manifestaron la necesidad de reforzar, en las llamadas, la forma de establecer mecanismos para contar con una medición efectiva de la cantidad de líquidos consumidos, así como para reconocer los signos de alarma en cuanto al control de peso, aparición de edema, signos de dificultad respiratoria o aumento de la tensión arterial.

\section{La actividad fisica, clave del tratamiento. El} proceso de intervención y acompañamiento resaltó la articulación de la tríada adherencia a los medicamentos - actividad física - hábitos alimenticios como ejes para el control sostenido de la EC.

Al respecto, cerca de la mitad de personas intervenidas refieren la importancia de la actividad física, planteando de manera explícita diversas estrategias como caminatas diarias, repeticiones de subir y bajar escaleras en el hogar y ejercicios isotónicos. Tres casos, además, solicitaron a su Empresa Promotora de Salud (EPS) la atención por parte de terapia física en el hogar. 
ISSN-PRINT

1794-9831

E-ISSN 2322-7028

Vol. 15 No. 1

Ene - Jun 2018

Cúcuta, Colombia
4. Medicamentos: alta adherencia versus baja compresión de horarios, interacciones y signos de alarma. Al realizar preguntas sobre el detalle del consumo de medicamentos (horarios, interacciones, signos de alarma) se encontró que los casos no contaban con claridades respecto de por qué se sugería tomar los medicamentos en horas específicas, si era antes o después de la alimentación y qué signos o síntomas podrían indicar el desarrollo de efectos adversos en relación con los anticoagulantes y las insulinas.

5. Dieta, restricciones en el consumo de sal y grasa. Los 37 casos manifestaron haber hecho ajustes, antes y después de la intervención, en relación con la moderación del consumo de sal y grasa. No obstante, el tema de consumo de frutas y verduras se observó limitado en la mayoría de participantes.

Adicionalmente, se evidenciaron bajos niveles de conocimiento en relación con la restricción del consumo de proteínas (en el paciente con enfermedad renal), bajos niveles de conocimiento en relación con el consumo de carbohidratos y glucosa en el resto de los casos y baja comprensión sobre la importancia de fraccionar los alimentos en mínimo 5 raciones diarias, temática que se debió abordar en varias llamadas telefónicas.

6. Demanda de aspectos emocionales, sociales $y$ económicos en el proceso de valoración e indicaciones. Los 37 casos reconocen que la EC les genera carga emocional importante, así como dificultades económicas que deben ser atendidas con ayuda de sus familiares. Es de anotar que no reconocen redes de apoyo social, ni cómo acceder a organizaciones o grupos de soporte.

La llamada telefónica facilitó la expresión de sentimientos, la verbalización de preocupaciones como la estabilidad laboral, el impacto económico de la incapacidad y la desocupación.

\section{Discusión}

El objetivo del estudio fue determinar el efecto de la intervención-acompañamiento telefónico sobre la competencia para el cuidado, así como las percepciones de los casos frente a la intervención. Los resultados revelan que hay incrementos estadísticamente significativos, hallazgos que coinciden con un estudio previo realizado con mayor énfasis en la educación brindada en el hospital (21).
El estudio reporta un aumento significativo de los componentes de conocimiento, unicidad y bienestar después de laintervención telefónica. Estas dimensiones exploran conocimientos básicos sobre la enfermedad, aspectos básicos de la vida cotidiana, establecimiento de un plan de vida, satisfacción personal relacionada con la calidad de vida percibida y manejo de sentimientos de culpa y frustración, hallazgos que coinciden con otros $(22$ - 24) que muestran que la consulta telefónica realizada por enfermería tiene impacto en el abordaje de esferas emocionales.

En vista de lo anterior, puede afirmarse que el esquema de soporte telefónico aquí propuesto tiene como resultados posibles: 1) Mejorar la calidad de vida percibida de personas que viven con enfermedad crónica; 2) Mejorar la calidad percibida sobre la prestación de los servicios de salud y facilitar la vinculación-adherencia de los usuarios a otro tipo de programas de promoción de la salud (programas de cuidado a pacientes crónicos, programas de cuidado a cuidadores, programas de mutua ayuda); 3) Proveer herramientas para el auto-manejo de la situación de cronicidad, desde una perspectiva compleja y contextualizada al estilo de vida y los recursos personales de cada usuario; 4) Crear escenarios de atención no-presencial que facilitan una comunicación más horizontal entre el equipo de enfermería y el usuario, lo que permite la expresión de preguntas y preocupaciones particulares.

Aunque aumentaron los niveles de competencia en los componentes instrumental y anticipación, estos cambios no son estadísticamente significativos, situación que puede estar asociada con la necesidad de complementar con otras tecnologías y/o piezas educativas el refuerzo de temas que comprenden el desarrollo de habilidades en manejo de medicamentos, reconocimiento de signos de alarma y predicción de situaciones de riesgo.

Además, este panorama puede ser indicativo de la necesidad de generar estrategias educativas específicas por tipo de patología, con instrucciones puntuales a dar en el alta y para reforzar en el seguimiento telefónico. Este último hecho representa un reto para la investigación y la práctica de enfermería, pues señala la necesidad de explorar la cronicidad no sólo como situación clínica, sino como experiencia personal frente a una patología(s) en particular, que 
demanda habilidades procedimentales y recursos psicosociales particulares.

La intervención telefónica implicó reconocer la vivencia subjetiva de quien experimenta la 'carga' de la enfermedad crónica. Decir que se afecta la vida cotidiana es entender que se perturba el conjunto de valores, orientaciones, actitudes, expectativas -sobre todo-, normas, conductas y práctica sociales.

Sin embargo, indagar en una consulta telefónica por aspectos emocionales, familiares, laborales y sexuales son aspectos que van más allá del diligenciamiento de un formato o que conforman los antecedentes de la historia clínica; hacen parte de 'un' comprender para actuar con la mayor asertividad posible y se relacionan, además, con el establecimiento de un vínculo. Esto es efectividad clínica.

Sin duda, profundizar en las implicaciones de la cronicidad implica pensar en la necesidad de evaluar lo cotidiano como una forma adecuada de análisis clínico (25). Este hecho, a su vez, plantea interrogantes sobre la formación y actualización de los equipos de enfermería frente al tránsito hospitalhogar-hospital, relativamente frecuente en algunas personas con enfermedad crónica.

Paradójicamente, producto de la revisión de literatura realizada, se encontró que dichas dimensiones no suelen incluirse en planes de cuidado, estrategias institucionales o como parte del tratamiento en sí (25). Los individuos en situación de cronicidad están devaluados, carecen de roles sociales y son de preferencia invisibles para el resto de la sociedad (26).

Ahora bien, la intervención telefónica plantea retos para enfermería en la búsqueda de respuestas a las personas con enfermedad crónica, lo cual implica una reorientación del tradicional modelo de atención curativa, caracterizado por los contactos episódicos y centrados en procesos agudos o descompensaciones hacia un diagnóstico integral que, además de describir los procesos clínicos, incluya su situación social, su contexto familiar y su grado funcional; una intervención más centrada en actuaciones esperanzadoras y rehabilitadoras para evitar su progresión que en sólo esfuerzos curativos, y, por supuesto, una implicación activa del paciente y su entorno familiar en la adherencia al tratamiento y aplicación de cuidados (3).

Por otro lado, la intervención plantea la necesidad de cuestionarnos sobre el efecto de la información entregada. Es sabido que la incertidumbre frente a la enfermedad se describe como la incapacidad de las personas para dar sentido o significado a los procesos o las situaciones que tienen que ver con su estado de salud, lo que se traduce en una imposibilidad para predecir los resultados y manejarlos, generalmente producida por la falta de información y conocimiento sobre la enfermedad.

No obstante, muchas veces el conocimiento sobre una situación genera más incertidumbre relacionada con la continua amenaza de desequilibrio o de revivificación de una situación de riesgo o daño, lo que lleva a diversas formas de afrontamiento (27).

En tal sentido, el alcance de las "llamadas telefónicas" va -y debe ir- más allá de brindar información sobre la enfermedad (28-30); se requiere el posicionamiento del cuidado de enfermería más allá del refuerzo de la prescripción médica, la integración efectiva con el equipo multidisciplinario para el abordaje oportuno de hallazgos, la integración de perspectivas de vida y muerte digna y la intervención sobre acompañantes y cuidadores.

Frente a las limitaciones del estudio, se precisa que el efecto de esta intervención necesita ser probado en un mayor número de pacientes, determinando el alcance de esta sobre otras variables de interés clínico sostenibles en el tiempo. Se requiere, además, explorar la adherencia a las terapéuticas que se reflejen en la práctica real.

Por otro lado, es necesario profundizar en el perfil de los profesionales de enfermería requeridos para este tipo de intervenciones, los costos de la implementación de la estrategia versus la disminución de reingresos hospitalarios por complicaciones prevenibles y la menor consulta a servicios de urgencias en las instituciones que prestan los servicios de salud.

Así mismo, es necesario generar estrategias que fortalezcan la adherencia a la propuesta de seguimiento telefónico por parte de las personas con EC en este tipo de intervenciones. 
ISSN-PRINT

1794-9831

E-ISSN 2322-7028

Vol. 15 No. 1

Ene - Jun 2018

Cúcuta, Colombia

\section{Conclusiones}

- La intervención telefónica aporta en la continuidad de la relación entre la persona con enfermedad crónica y el sistema de salud. Se reconoce, además, sus efectos sobre la competencia para el cuidado en el hogar. Se consolida como una estrategia que brinda soporte emocional, facilita la retroalimentación y la puesta en común de inquietudes que surgen en los usuarios una vez egresan del hospital.

- La facilidad de acceso, la disponibilidad y los costos se plantean como variables favorables a tener en cuenta para su implementación en diferentes instituciones de salud. Así mismo, contar con un protocolo de seguimiento acorde con las posibilidades culturales de los pacientes con enfermedad crónica refleja respuestas esperadas por parte de enfermería en la implementación del Modelo Integral de Atención en Salud (MIAS).

\section{Conflicto de Intereses}

Las autoras declaran no tener ningún conflicto de intereses.

\section{Referencias Bibliográficas}

1. Organización Panamericana de la salud. Detener la epidemia mundial de enfermedades crónicas: una guía práctica para la promoción exitosa de la causa [Internet] 2016. [consultado el 2/09/2017]. Disponible en: https://goo.gl/zAob7U.

2. Organización Mundial de la Salud. Informe sobre la situación mundial de las enfermedades no transmisibles [Internet] 2014. [Consultado el 8/08/2017]. Disponible en: https://goo.gl/BnsPP6.

3. Melguizo Jiménez M. De la enfermedad crónica al paciente en situación de cronicidad. Aten Primaria 2011; 43:67-8.

4. Administración de la Comunidad Autónoma del País Vasco Departamento de Sanidad y Consumo de Sanidad y Consumo [Internet] 2008. [Consultado el 5/07/2017]. País Vasco: transformando el Sistema de Salud 2009 - 2012. Disponible en: https://goo.gl/4sBSWr.

5. Barrera Ortiz L, Blanco de Camargo L, Figueroa P, Pinto Afanador N, Sánchez Herrera B. La habilidad de cuidado de los cuidadores familiares de personas con enfermedad crónica. Aquichan 2009; 23 (1): 36-46.

6. Pinto Afanador N. Comprender la situación de cronicidad que viven nuestros pacientes. En: Grupo de Cuidado. La investigación y el cuidado en América Latina. Bogotá: Universidad Nacional de Colombia, Facultad de Enfermería, Unibiblos; 2004.

7. Organización Mundial de la Salud, Consejo Ejecutivo. Formulación de estrategias con el fin de alcanzar la salud para todos en el año 2000: principios básicos y cuestiones esenciales [Internet] 1979 [consultado el 2/07/2017]. Disponible en: https://goo.gl/fq68Yg.

8. Vinaccia S, Orozco LM. Aspectos psicosociales asociados con la calidad de vida de personas con enfermedades crónicas. Diversitas Bogotá. 2005; 1(2):125-37.

9. Ledón Llanes, L. Enfermedades crónicas y vida cotidiana. Rev. Cubana Salud Pública. 2011 Dic; 37(4): 488-499.

10. Medina O. Encuentro con la cronicidad. Rev. Desarrollo Cientif. Enferm. 2003; (11): 79-82.

11. Vargas Escobar LM. Marco para el cuidado de la salud en situaciones de enfermedad crónica. Investigación en Enfermería: Imagen y Desarrollo. 2010; 12(1):79-94.

12. Ministerio de Salud y Protección Social, Colombia. Política de atención integral en salud: "Un sistema de salud al servicio de la gente" [Internet] 2016. [consultado el 25/05/2017]. Disponible en: https:// goo.gl/9y9SwL.

13. Dadgari F, Hoseini S, Aliyari S, Masoudi S. The effect of sustained nursing consulting via telephone (Tele nursing) on the quality of life in hypertensive patients. Applied Nursing Research 2017; 35(6):106-111. 
14. Muller C, Lautenschlager S, Meyer G, Stephan A. Interventions of support people with dementia and their caregivers during the transition from home care to nursing home care: A systematic review. International Journal of Nursing Studies 2017; 71:139-152.

15. Shearer, NB, Cisar, N, Greenberg, EA. A telephone-delivered empowerment intervention with patients diagnosed with heart failure. Heart \& Lung 2007; 30, 159-169.

16. Ajalli, A, Fallahi Khoshknab, M. Tele nursing care in chronic patients/a systematic review. Iranian Journal of Rehabilitation Research in Nursing 2015;1, 76-86.

17. Polit D, Hungler B. Investigación científica en ciencias de la salud. 6ta ed. México: McGraw-Hill Interamericana. 2000; p. 241.

18. Carrillo González GM, Sánchez Herrera B, Vargas Escobar LM. Desarrollo y pruebas psicométricas del Instrumento "cuidar" - versión corta para medir la competencia de cuidado en el hogar. Rev. Univ. Ind. Santander. Salud. 2016; 48(2):222-231.

19. Consejo de Organizaciones Internacionales de las Ciencias Médicas CIOMS. Pautas éticas internacionales para la investigación Biomédica en seres humanos [Internet]. 2002. [Consultado el 8/01/2016]. Disponible en: https://goo.gl/LL6XsM.

20. Resolución 008430 de 1993. Por la cual se establecen las normas científicas, técnicas y administrativas para la investigación en salud. Ministerio de Salud y Protección Social, Colombia [Internet] 1993 [Consultado el 6/03/2016]. Disponible en: https://goo.gl/aAdihN.

21. Melo B, Carrillo González GM, Vargas Y, Alarcón D. Efecto del programa plan de egreso en la diada persona con enfermedad crónica y sus cuidadores familiares. Enfermería clínica. 2017 En prensa.

22. Frasure Smith N, Lesperance F. Depression and anxiety as predictors of 2 year cardiac events in patients with stable coronary artery disease. Archives of General Psychiatry. 2008; 65, 62-71.

23. Sabzmakan L, Hazavehei S, Morowatisharifabad M, Hasanzadeh A, Rabiee K, Sadeqi M. The effects of a PRECEDE-based educational program on depression, general health, and quality of life of coronary artery bypass grafting patients. Asian Journal of Psychiatry. 2010; 3, 79-83.

24. Babaee M, Keshawarz A, Hidarnia A. Effect of health education program on Quality of life in Evaluation of quality of life in patients with coronary artery parients undergoing coronary artery bypass surgery. Bypass surgery using controlled clinical trial. Acta Medica Iranica. 2007; 45, 69-75.

25. Mesa Melgarejo L, Carrillo Algarra AJ, Moreno Rubio F. La cronicidad y sus matices: estudio documental. Investigación en Enfermería: Imagen y Desarrollo. 2013; 15(2):95-114.

26. Zavala Rodríguez MR, Whetsell MV. La fortaleza de los pacientes con enfermedad crónica. Aquichan 2007 octubre: $174-188$.

27. Gómez Palencia IP, Castillo Ávila IY, Alvis Estrada LR. Incertidumbre en adultos diabéticos tipo 2 a partir de la teoría de Merle Mishel. Aquichan. 2015; 15(2):210-218.

28. Padilla Just MN, Ros del Hoyo N, Raventós Castany A, Escribano Rueda V, etal. Seguimiento telefónico enfermero a pacientes con insuficiencia cardiaca. Metas de Enferrn abr 2011; 14(3):22-26

29. Bejarano DF, González N, Lozano LM, Pinto N. Seguimiento al plan de egreso hospitalario con uso de tecnologías de la información y la comunicación (teléfono fijo-móvil y/o mensajes de texto) a cuidadores familiares de niños con enfermedad crónica en el Instituto de Ortopedia Infantil Roosevelt. Investigación en Enfermería: Imagen y Desarrollo. 2011; 13(1):93-105.

30. Romero S, Villamizar B, Rueda L. Apoyo telefónico: Una estrategia de intervención para cuidadores familiares de personas con enfermedad crónica. Salud UIS 2011; 43(2):191-201. 